\title{
Homonymies in Callichromatini (Coleoptera, Cerambycidae) ${ }^{1}$
}

\author{
Dilma Solange Napp ${ }^{2,4}$ \& Ubirajara R. Martins $s^{3,4}$
}

\begin{abstract}
${ }^{1}$ Contribution $\mathrm{n}^{0} 1593$ of the Departamento de Zoologia,Universidade Federal do Paraná.
${ }^{2}$ Departamento de Zoologia, Universidade Federal do Paraná. Caixa Postal 19020, 81531-980 Curitiba-PR, Brazil.

${ }^{3}$ Museu de Zoologia, Universidade de São Paulo. Caixa Postal 42494, 04218-970 São Paulo-SP, Brazil.

${ }^{4}$ Pesquisador do $\mathrm{CNPq}$
\end{abstract}

\begin{abstract}
Homonymies in Callichromatini (Coleoptera, Cerambycidae). New names proposed: Beaveriella nom. nov. for Beaveria Lane, 1970, pre-occupied by Beaveria Lee, 1965 (Trematoda, Troglotrematidae); Monnechroma nom. nov. for Xenochroma Schmidt, 1924, pre-occupied by Xenochroma Warren, 1902 (Lepidoptera, Geometridae). Consequently, the following new combinations are made: Beaveriella nacta (Lane, 1970) comb. nov., B. sagda (Monné \& Martins, 1972) comb. nov., Monnechroma azurea (Demets, 1976) comb. nov., M. seabrai (Fragoso \& Monné, 1989) comb. nov., M. subpulvereum (Schimidt, 1924) comb. nov., M. tibialis (Giesbert, 1987) comb. nov., and M. uniforme (Gounelle, 1911) comb. nov..
\end{abstract}

KEYWORDS. Beaveriella; Cerambycinae; Monnechroma; nomenclatural changes; taxonomy.

RESUMO. Homonímias em Callichromatini (Coleoptera, Cerambycidae). São propostos: Beaveriella nom. nov. para Beaveria Lane, 1970, pré-ocupado por Beaveria Lee, 1965 (Trematoda, Troglotrematidae); Monnechroma nom. nov. para Xenochroma Schmidt, 1924, pré-ocupado por Xenochroma Warren, 1902 (Lepidoptera, Geometridae). Conseqüentemente, as seguintes novas combinações são efetuadas: Beaveriella nacta (Lane, 1970) comb. nov., B. sagda (Monné \& Martins, 1972) comb. nov., Monnechroma azurea (Demets, 1976) comb. nov., M. seabrai (Fragoso \& Monné, 1989) comb. nov., M. subpulvereum (Schimidt, 1924) comb. nov., M. tibialis (Giesbert, 1987) comb. nov. e M. uniforme (Gounelle, 1911) comb. nov..

PALAVRAS-CHAVE. Beaveriella; Cerambycinae; Monnechroma; nomenclatura; taxonomia.

\section{Beaveriella nom. nov.}

Beaveria Lane, 1970: 51 (non Beaveria Lee, 1965); Monné, 2005: 24 (cat.).

Type species: Beaveria nacta Lane, 1970, by original designation.

Lane (1970) described Beaveria as a genus of Coleoptera, Cerambycidae, Cerambycinae, Callichromatini. However, this name was used by Lee (1965) in Trematoda, Troglotrematidae. It is here proposed, Beaveriella, as a substitution name. As a result, the following changes are introduced:

\section{Beaveriella nacta (Lane, 1970) comb. nov.}

Beaveria nacta Lane, 1970: 52; Monné, 2005: 24 (cat.).

Beaveriella sagda (Monné \& Martins, 1972) comb. nov. Beaveria sagda Monné \& Martins, 1972: 76, fig. 1; Monné, 2005: 24 (cat.).

\section{Monnechroma nom. nov.}

Callichroma (Xenochroma) Schmidt, 1924: 300, 378 (non Xenochroma Warren, 1902).

Xenochroma; Podany, 1965: 38; Monné, 2005: 38 (cat.).

Type species: Callichroma (Xenochroma) subpulvereum Schmidt, 1924, by original designation.

Schmidt (1924) proposed Xenochroma to designate a subgenus of Callichroma (Coleoptera, Cerambycidae, Cerambycinae). However, this name was found to be pre- occupied by Xenochroma Warren (1902) in Lepidoptera, Geometridae. Therefore, Monnechroma nom. nov., is here proposed. The nominal genus is in honor to Miguel A. Monné. The following changes are introduced:

Monnechroma azurea (Demets, 1976) comb. nov. Xenochroma azurea Demets, 1976: 136; Monné, 2005: 38 (cat.).

Monnechroma hovorei (Giesbert, 1998) comb. nov.

Xenochroma hovorei Giesbert, 1998: 62, fig. 3; Monné, 2005: 38 (cat.)

Monnechroma seabrai (Fragoso \& Monné, 1989) comb. nov. Xenochroma seabrai Fagoso \& Monné, 1989: 210; Monné, 2005: 38 (cat.).

Monnechroma subpulvereum (Schmidt, 1924) comb. nov. Callichroma (Xenochroma) subpulvereum Schmidt, 1924: 309. Xenochroma subpulvereum; Podany, 1965: 39; Monné, 2005: 38 (cat.)

Monnechroma tibialis (Giesbert, 1987) comb. nov. Xenochroma tibialis Giesbert, 1987: 36, fig. 1; Monné, 2005: 38 (cat.).

Monnechroma uniforme (Gounelle, 1911) comb. nov. Callichroma uniforme Gounelle, 1911: 169.

Callichroma (Xenochroma) uniforme; Scmidt, 1924: 379.

Xenochroma uniforme; Podany, 1965: 39; Monné, 2005: 38 (cat.) 
Acknowledgment. We thank Dr. Miguel A. Monné who discovered the homonymies and provided us the references.

\section{REFERENCES}

Demets, Y. 1976. Notes sur les Callichromatini (Coleoptera, Cerambycidae). IV. Étude preliminaire des genres Schwarzerion Schmidt, 1924 et Xenochroma Schmidt, 1924. Papéis Avulsos de Zoologia 29: 121-140.

Fragoso, S. A. \& Monné, M. A. 1989. Duas novas espécies de Callichromatini neotropicais (Coleoptera, Cerambycidae, Cerambycinae). Memórias do Instituto Oswaldo Cruz 84 (suppl. IV): 209-212.

Giesbert, E. F. 1987. Additions to the Callichromatini of Central America, with a key to genera and description of a new species of Xenochroma (Coleoptera: Cerambycidae). The Coleopterists Bulletin 41: 35-40.

Giesbert, E. F. 1998. Further studies in the Neotropical Callichromatini (Coleoptera, Cerambycidae, Cerambycinae). Occasional Papers of the Consortium Coleopterorum 2: 60-64.

Gounelle, E. 1911. Description de quelques espèces nouvelles de Callichroma du Brésil méridional (Col., Cerambycidae). Bulletin de la Société Entomologique de France 1911: 165-170.

Lane, F. 1970. Beaveria nacta, gen. n., sp. n. de Purpuricenini. Revista Brasileira de Entomologia 14: 51-55.

Lee, H-F. 1965. Digenetic Trematodes of Feral Rats from Malaysia with descriptions of Beaveria beaveri and B. microacetabulum gen. n., spp. n., of a New Subfamily Beaveriinae (Troglotrematidae). The Journal of Parasitology 51: 24-29.

Monné, M. A. 2005. Catalogue of the Cerambycidae (Coleoptera) of the Neotropical Region. Part I. Subfamily Cerambycinae. Zootaxa 946: $1-765$.

Monné, M. A. \& U. R. Martins. 1972. Sôbre Triacetelus Bates e Beaveria Lane (Coleoptera, Cerambycidae, Purpuricenini). Revista Brasileira de Entomologia 16: 75-78.

Podany, C. 1965. Remarques sur les Callichromini américains (Col., Ceramb.). Bulletin de la Société Entomologique de Mulhouse 1965: $34-41$.

Schmidt, M. 1924. Die amerikanischen Callichrominen (Col., Ceramb.) nach systematischen und phylogenetischen Gesichtspunkten dargestellt. Deutsche Entomologishe Zeitschrift 1924: 297321; 377-396.

Warren, W. 1902. New African Drepanulidae, Thyrididae, Epiplemidae and Geometridae in the Tring Museum. Novitates Zoologicae 9: $487-536$. 